\title{
Development and assessment of the psychometric properties of a new scale (15 item PSB-CL) to monitor the psychosocial burden of chronic lymphoedema in filariasis
}

\author{
Ruben $\mathbf{J}^{1}$, Yahathugoda $\mathrm{TC}^{1}$, Weerasooriya $\mathbf{M V}^{1}$, Punchihewa $\mathbf{M W}^{2}$, Senadheera $\mathbf{C}^{3}$, Somasiri $\mathbf{K G}^{4}$, \\ Perera B ${ }^{5}$ \\ Departments of ${ }^{1}$ Parasitology, ${ }^{3}$ Psychiatry, ${ }^{4}$ Physiology, ${ }^{5}$ Community Medicine, Faculty of Medicine, University \\ of Ruhuna, Galle, Sri Lanka. \\ ${ }^{2}$ Anti Filariasis Campaign, Office of Regional Director Health Services, Galle, Sri Lanka.
}

Correspondence: Dr. Janaka Ruben

e-mail: janakaruben@med.ruh.ac.lk

(1) https://orcid.org/0000-0001-6391-8255

\begin{abstract}
Introduction: Chronic lymphoedema (CL) of lymphatic filariasis causes severe psychosocial burdens (PSB) to its victims. Currently-established scales to measure PSB of CL are not culturally adaptable to Sri Lankan patients. In this study, we developed and assessed the psychometric properties of a disease-specific questionnaire (15 item PSB-CL).

Methods and Results: A detailed literature search was done to identify scales related to PSB of CL patients. Questions were developed through collected expressions from semi-structured interviews with $46 \mathrm{CL}$ patients. A response scale was developed from the collected pool of possible responses, and they were scored using a visual analogue scale. Face and content validity were carried out using an initial questionnaire (31 questions). They were reduced to 27 by removing unclear, ambiguous, double-stranded ones and those with value-laden words. This new questionnaire was administrated on 92 patients by a group of doctors for item reduction by the analysis of internal consistency (Cronbach's alpha $\geq 0.7$ ) and factor analysis (Principal Component Analysis; Eigenvalues $\geq 1$ ). After the patient survey and factor analysis, the final questionnaire PSB-CL was formed with 15 questions that had four subscales (factors), physical, social, fear and humiliation. Patients ( $\mathrm{n}=92$ ) reported quite a large percentage $(42 \%-69 \%)$ of problems under each dimension.
\end{abstract}

Conclusion: The scale to measure the psychosocial burden of chronic lymphoedema in lymphatic filariasis patients in Sri Lanka. Further validation of the tool is needed.

Keywords: Lymphatic filariasis, measuring scale, psychosocial burden

\section{Introduction}

Lymphatic filariasis (LF) is a tropical disease caused by thread-like worms - Wuchereria bancrofti, Brugia malayi, Brugia timori - living in the human lymph system (1). LF is one of the leading neglected tropical diseases, which causes debilitating and disfiguring conditions (2). Lymphoedema (elephantiasis), acute episodes of adenolymphangitis (ADL), hydrocoele, lymph scrotum, and chyluria are due to the damage of lymphatic systems. Other symptoms such as arthritis, renal diseases, and tropical pulmonary eosinophilia (TPE) are also ascribed to extralymphatic filarial infection $(3,4)$. Acute episodes of ADL, lymphoedema and hydrocoele are the three principal manifestations of LF that cause severe suffering (5). 
It has been reported that more than one billion people are at risk of infection, of whom 120 million are infected in endemic countries before the establishment of the global programme to eliminate lymphatic filariasis (GPELF) in 2000 (6). The prevalence of lymphoedema and hydrocoele were estimated to be $3 \%$ and $6.2 \%$ respectively in the three suburbs of Matara, Sri Lanka (7). Previous estimates to the whole population at risk (10 million) were scaled up to give an expected 300,000 cases of lymphoedema and around 300,000 cases of hydrocoele in eight endemic districts of Sri Lanka (8). As for acute episodes of ADL, 46.6\% to $58.4 \%$ of lymphoedema patients have experienced ADL at least once in Sri Lanka (9-11).

There are several medical and surgical options developed recently to alleviate suffering from lymphoedema. Those include morbidity manage-ment and disability prevention (MMDP) with hygiene improvement, limb elevation and exercise, topical application of antibiotics/ antifungals to prevent infections and surgical resection (12-14).

MMDP programme is to alleviate suffering of individuals who are not fully capable of longterm self-care maintaining the near-normal life. Patients should reach the highest possible degree of independence, autonomy, participation and personal fulfilment by activities carried out at home by themselves or by formal/ informal carers (family, friends, neighbours, traditional carers and volunteers) (14).

However, psychological distress, social dysfunction and coping strategies of patients with chronic filariasis can hamper rehabilitation of the affected. The resumption of near 'normal life' of such patients becomes unreal. Studies carried out in the past by many scientists have documented the psychological burden associated with $\operatorname{LF}(8,11,15,16,17)$. Studies carried out in Sri Lanka specially recorded physical, mental and social suffering due to lymphoedema $(9,11,18)$.

Formulation and validation of a tool to assess the psychological burden of LF patients in Sri Lanka would be valuable to the current MMDP programme because the medical officers who are attached to primary health care settings may not have the capacity to identify psychological burden with ease. Therefore, incorporation of such a developed tool will facilitate the assessment of the psychosocial burden of LF patients in current MMDP programme (17-19). Its expression should be based on cultural norms and values and collection of information from the affected people without a preoccupied conceptual framework. Thus, the current study was aimed to develop a new scale to be used in local settings to identify the psychosocial burden of chronic lymphoedema in LF patients. In this article, only the development of the scale and assessment of properties are explained.

\section{Methods}

The PSB-CL questionnaire was designed based on standardised health care, health-related quality of life (HRQoL) questionnaire development, and validation methodology proposed by WHO (19). The questionnaire was developed in four phases; item generation, item selection, dimension reduction, response development and evaluation (19). Suitable responses to the questions were also developed and evaluated using participants living in the community. Appropriate phrases for the answers were formulated using focusgroup discussions with the patients. Thus, item development, item selection, item reduction, response development, response selection, question-naire formulation and content validation of the questionnaire were made during the first stage of the study. In the second stage, secondary question reduction was conducted using exploratory factor analysis to develop the final questionnaire (19).

Item development: A critical review of existing tools to measure the psychosocial burden of LF was carried out by a six-member committee comprised principal investigator, two senior parasitologists, a clinical psychologist, a behavioural science specialist and a physiologist.

None of the scales captured behaviour, feeling, or actions related to psychosocial dimensions of local people as we expected. Therefore, key informant interviews were planned as the source of items. A topic guide was developed by carrying out three in-depth interviews with local patients by the authors. The authors JR, CY, CS and BP have conducted in-depth interviews with the patients. Patients were selected from the patient databases 
at FRTSU, University of Ruhuna considering age, sex, grade of lymphoedema and social background (Table 1). Patients from endemic communities in Galle and Matara, Sri Lanka, were included in the databases. Interviews were audio-recorded and transcribed.

In-depth interviews lasted for 1.30 to 2.30 hours; more than half a day to transcribe one script. Patients were interviewed until no new themes emerged.
Careful analysis of transcriptions produced several dimensions of psychosocial burden of LF. A series of expressions related to such domains were categorised; some expressions placed in more than one dimension.

Table 1: Distribution of demographic data and the stages of the lymphoedema of participants $(\mathrm{n}=46)$

\begin{tabular}{|c|c|c|c|}
\hline \multirow[t]{4}{*}{ Age } & Mean & \multicolumn{2}{|l|}{49.61} \\
\hline & SD & \multicolumn{2}{|l|}{16.25} \\
\hline & \multirow[t]{2}{*}{ Range } & $18-80$ & \\
\hline & & No. & $(\%)$ \\
\hline \multirow[t]{2}{*}{ Sex } & Female & 34 & $(73.9)$ \\
\hline & Male & 12 & $(26.1)$ \\
\hline \multirow[t]{6}{*}{ Marital status } & Married & 31 & $(67.4)$ \\
\hline & Married but living separately & 2 & $(4.3)$ \\
\hline & Unmarried but living together & 2 & (4.3) \\
\hline & Unmarried & 7 & $(15.2)$ \\
\hline & Divorced & 1 & $(2.3)$ \\
\hline & Widowed & 3 & $(6.5)$ \\
\hline \multirow[t]{5}{*}{ Education } & No formal education & 3 & $(6.5)$ \\
\hline & Up to primary education & 14 & $(30.4)$ \\
\hline & up to $\mathrm{O} / \mathrm{L}$ & 9 & $(19.6)$ \\
\hline & up to $\mathrm{A} / \mathrm{L}$ & 17 & $(37.0)$ \\
\hline & University degree & 3 & $(6.5)$ \\
\hline \multirow[t]{2}{*}{ Occupation } & Employed & 22 & $(47.8)$ \\
\hline & Unemployed & 24 & $(53.2)$ \\
\hline \multirow[t]{4}{*}{ Monthly income } & $<$ Rs. $10,000 /=$ & 1 & $(2.3)$ \\
\hline & Rs. $10,001 /=$ to $20,000 /=$ & 27 & $(58.7)$ \\
\hline & Rs. $20,001 /=$ to $30,000 /=$ & 14 & $(30.4)$ \\
\hline & $>$ Rs. $30,001 /=$ & 4 & $(8.6)$ \\
\hline \multicolumn{4}{|l|}{ Lymphoedema } \\
\hline \multirow[t]{4}{*}{ Grade } & Grade I & 3 & $(6.5)$ \\
\hline & Grade II & 20 & $(43.5)$ \\
\hline & Grade III & 16 & $(34.8)$ \\
\hline & Grade IV and above & 7 & $(15.2)$ \\
\hline
\end{tabular}


Item selection: Transcripts were analysed using a systematic approach to thematic analysis based on principles of the grounded theory which is an inductive approach aiming to derive theoretical construction by identifying repeated themes (repeating ideas) in transcripts (21). Selected items were pre-tested to ensure that local patients understood them; that they were unambiguous and the questioned only once. A Likert-type scale ranging from 1 (not clear/not frequent/not important) to 5 (very clear/ frequent/ important) was prepared to pre-test the questionnaire. Each expression was assessed by experts (authors, including the principal author) and given a grading to the Likert-chart. The grading of selected items was analysed by assessing the degree of concordance among experts using Cronbach's alpha (Table 2). Finally, a qualitative reduction of the items was carried out based on the Cronbach's alpha derived from the experts' responses. Not clear/ not frequent/ not important questions were rewritten and pre-tested again using the same methodology. Discarded items were not endorsed by many as important.

\section{Responses development and response selection:} The responses expressed the level of agreement for the intensity or frequency of the patients' problem generated through the patient's transcriptions (20). Out of response pool, two anchor points were then selected as "Not at all" considered getting zero points while "Extremely or Every time" got 100 points. The group of patients $(n=30)$ and research experts $(n=9)$ asked to score for the given responses between zero and 100 using a visual analogue scale $(100 \mathrm{~cm})$. Visual analogue scale scores were analysed according to the frequency placed consecutively in between end responses. Responses showing inconsistency were discarded.

Selected responses were coupled with questions in the most suitable positions. However, depending on some questions (whether they were measuring frequency, intensity or duration of the problem) if there were two responses with similar score, they had to be placed in the same positions to facilitate comprehension of questions.

Repeat pre-testing and face validity: The preliminary questionnaire that consisted of 31 (Table 2) questions was administrated to a group of 20 patients by the principal investigator. Administrative problems, patients' response errors and other difficulties were documented. To assess the administrative issues, the clinical practitioner was asked to simply rate each item on a five-point Likertscale ranging from Extremely Suitable to Irrelevant (22).

Reduction of attributes: The first stage of question reduction was completed, considering the results of the face validation phase. The internal consistency of the scale was analysed using Cronbach's alpha (23) to eliminate one attribute at a time. Item validation was done using item-total correlation and eliminated if the Pearson's $r$ was less than 0.200. We then ranked all attributes starting with the highest correlation. Cronbach's alpha coefficient of $\geq 0.700$ was considered satisfactory (Table 2).

Factor analysis: The final questionnaire (15 attributes - Table 3) was administered by trained pre-intern medical doctors and the principal investigator to patients from Galle and Matara districts. Sample adequacy was measured using Kaiser-Mayer-Olkin test. The data set was analysed using principal component analysis by having direct Oblimin rotation. However, Pattern Matrix showed none of the convergence value was greater or equal to 0.32 . Therefore Varimax rotation with Kaiser Normalization was used in the factor analysis using SPSS software to evaluate possible factors (themes) of PBS-CL. Based on eigenvalue (1 or more), factors were removed from the PBS-CL scale.

\section{Results}

Item development, content validity and item reduction

Forty-six (46) in-depth interviews were conducted. The mean age of the sample subjects was 49.6. Table data are summarised in Table 1.

Five (05) major dimensions related to psychosocial burden of patients were identified through the coding process. Expressions related to the identified dimensions were then recognised. Some expressions were placed in more than one dimension. Five major dimensions were i) restriction in social 
activities due to lymphoedema, ii) limitation of physical activities due to the lymphoedema, iii) fear of worsening the disease condition and deterioration of disability in the future, iv) the extent to which they were humiliated due to the lymphoedema condition, v) actions and thoughts related to psychological defence mechanisms.

In the process of face validation, 31 expressions were identified from the primary 48 expressions by removing ambiguous, complex questions and questions with value-laden words and jargon terms. They were transformed into statements (attributes) (Table 2).
Eight patients (08) out of twenty (20) described some questions as difficult to answer and requested further clarification. Four (04) such questions were removed from the preliminary questionnaire with 31 attributes. Following content validity, the expert panel has identified 27 attributes (Table 2).

Response development and response selection: Intensity measuring responses were developed as, (a) Not at all (b) to some extent (c) usually (d) on most occasions (e) extremely and frequency measuring responses were developed as (a) Not at all (b) sometimes (c) usually (d) often (e) every time.

Table 2: Item reduction by face and content validity (extremely suitable [100\%] or irrelevant $[<25 \%]$ ) and by internal consistency assessment (Cronbach's alpha $\geq 0.700$ )

\begin{tabular}{|c|c|c|c|}
\hline & Attributes & $\begin{array}{l}\text { Relevancy } \\
\text { \& Clarity }\end{array}$ & $\begin{array}{l}\text { When } \\
\text { removed } \\
\text { alpha is } \\
=0.700\end{array}$ \\
\hline 1 & Has your diseased condition affected adversely to your day to day activities? & $100 \%$ & No \\
\hline 2 & Do you experience any discomfort during travelling as a result of this disease condition? & $100 \%$ & No \\
\hline 3 & Do you get the assistance of the others in attending to your work as a result of this disease condition? & $100 \%$ & No \\
\hline 4 & Do you believe that this disease is your fate or an inevitable condition? & $50 \%$ & No \\
\hline 5 & Are you disgusted or frustrated with your life as a result of this disease condition? & $100 \%$ & No \\
\hline 6 & Do you feel sorry for yourself when you see person hale and hearty? & $100 \%$ & No \\
\hline 7 & Do you have any fear that this disease will worsen further? & $100 \%$ & No \\
\hline 8 & Do you have any fear that you would become disabled for long? & $100 \%$ & No \\
\hline 9 & Have you required to design or choose clothes, especially to suit you as a result of this disease? & $50 \%$ & No \\
\hline 10 & How much this disease is troublesome to you when considering your health and other issues? & $100 \%$ & No \\
\hline 11 & Do you have worries that other members of your family will get infected with this illness through you? & $100 \%$ & No \\
\hline 12 & Do you try to keep this diseased condition as a secret from the others? & $100 \%$ & No \\
\hline 13 & Do you feel ashamed whenever your neighbours see your diseased condition? & $100 \%$ & No \\
\hline 14 & Do you think your family members will feel ashamed as a result of your diseased condition? & $100 \%$ & No \\
\hline 15 & Do you have difficulties in sitting or standing due to this diseased condition? & $100 \%$ & No \\
\hline 16 & Do you suffer from intermittent fever as a result of this disease condition? & $100 \%$ & No \\
\hline 17 & Do you suffer from pain as a result of this disease condition? & $100 \%$ & No \\
\hline 18 & Do you get satisfaction, relief by chatting and keeping company with your friends? & $25 \%$ & Yes \\
\hline 19 & Do you feel overburden with your day to day work? & $50 \%$ & No \\
\hline 20 & When you feel worried, do you get a consolation in reading books, newspapers or magazines? & $25 \%$ & Yes \\
\hline 21 & Do you feel that you are an inconvenience to others as a result of this disease? & $25 \%$ & Yes \\
\hline 22 & Do you feel reluctant to get treatment for your diseased condition? & $25 \%$ & Yes \\
\hline 23 & Do you feel relieved considering that there are patients with more severe disease than you? & $25 \%$ & Yes \\
\hline 24 & Do you feel calm by involving in religious activities whenever you feel troubled by this diseased condition? & $25 \%$ & Yes \\
\hline 25 & When you feel worried, do you get comfort by watching television? & $25 \%$ & Yes \\
\hline 26 & How do you feel the way your family members treat you? & $25 \%$ & Yes \\
\hline 27 & Do you suffer more from other illnesses than this disease condition? (E.g., Diabetes, Hypertension) & $25 \%$ & Yes \\
\hline 28 & Do you often think and sob about your illness? & $25 \%$ & Yes \\
\hline 29 & Do you blame yourself or anyone else about your illness? & $25 \%$ & Yes \\
\hline 30 & Are you conversing with your family or friends about your illness? & $25 \%$ & Yes \\
\hline 31 & Do you feel uncomfortable during sexual activities as a result of this disease condition? & $50 \%$ & No \\
\hline
\end{tabular}




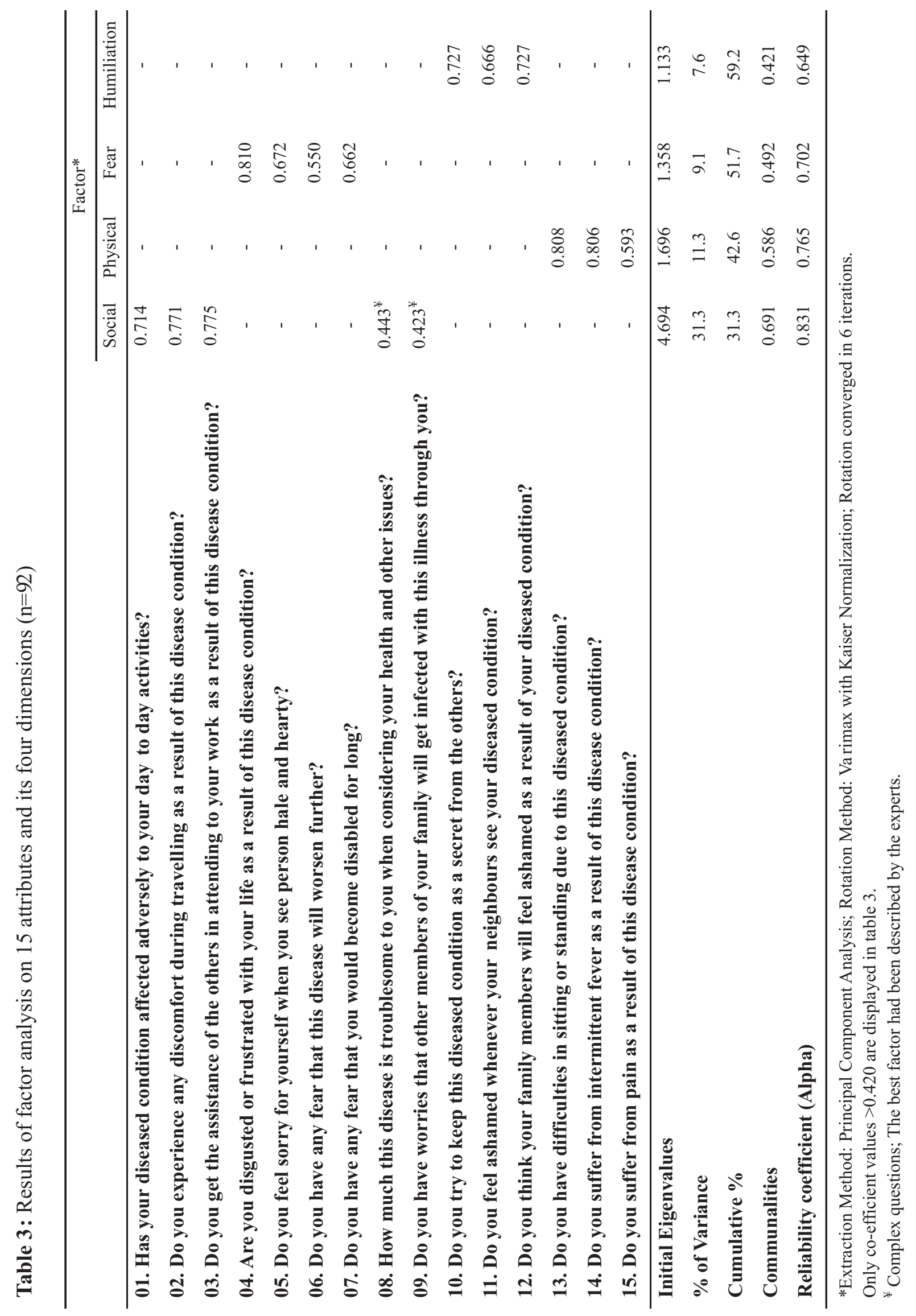




\section{Reduction of attributes and finalising response scale}

Cronbach's alpha was $0.765 \quad(n=90)$ for the questionnaire of 27 attributes. Had nine (09) attributes were removed, the Cronbach's alpha was greatly improved. Removal of another three (03) attributes would not have changed Cronbach's alpha. Considering all these facts, a final questionnaire with 15 attributes was constructed. The response scale was modified as (a) not at all (b) sometimes (c) usually (d) on most occasions (e) every time/ always, to suit to all 15 attributes.

\section{Factor analysis}

Kaiser-Meyer-Olkin Measure of sampling adequacy was 0.832. Bartlett's Test of Sphericity was $\chi^{2}=391.9 ; \mathrm{df}=105 ; \mathrm{p}<0.0001$. Four (04) factors have been extracted from the data in factor analysis of the 15 attributes (Table 3 ).
All factors identified in conjunction, accounted for $59.2 \%$ of the variance explained, the first factor had a variance explained of $31.3 \%$, second of $11.3 \%$, third of $9.1 \%$ and $7.6 \%$ for the fourth factor. The remaining eleven (11) factors had less than $5 \%$ of variance explained. The first factor consists of five (05) attributes, and others have 3 to 4 attributes. Item allocation to four factors was done when the item load was above 0.40 . However, two attributes had recorded item load $\geq 0.40$ in more than one-factor (Table 3). Experts described those complex attributes under a most suitable factor. None of the attributes were removed after factor analysis.

The analysis of PSB-CL questionnaire scores and its dimensions in terms of age, sex etc. is out of the scope of this part of the study. However, mean Likert scores under each dimension had shown a quite large percentage $(42 \%-69 \%)$ of patients having problems under four dimensions described above (Figure 1).

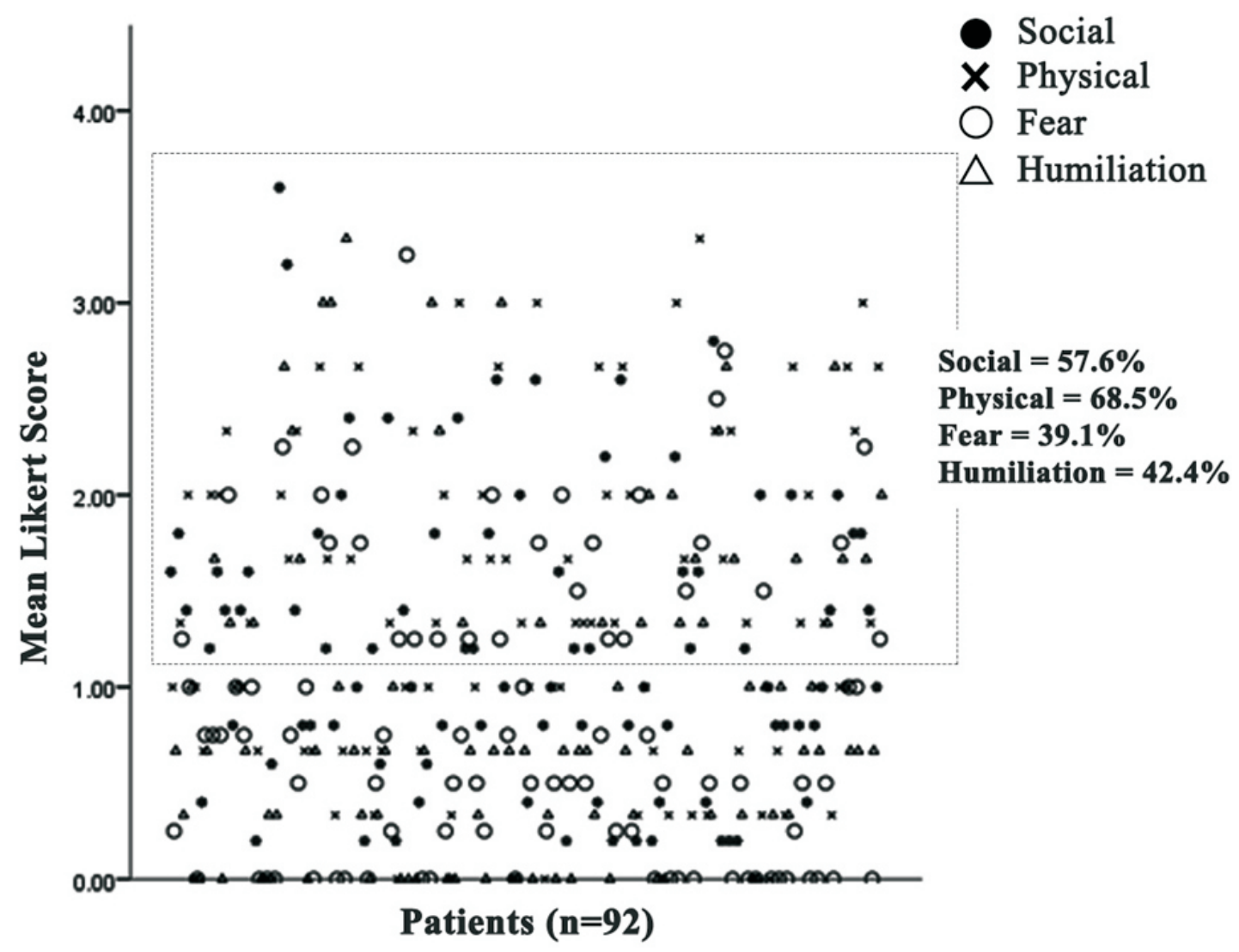

Figure 1: PSB-CL mean Likert score of four dimensions - Social, Physical, Fear and Humiliation $(\mathrm{n}=92)$ 


\section{Discussion}

The newly developed health measurement scale, 15 item PSB-CL has shown promising results in the primary psychometric analysis (significant internal consistency Cronbach's alpha $\geq 0.8$ and factor analysis-four meaningful dimensions). It will measure the psychosocial burden of chronic filarial lymphoedema patients in Southern Sri Lanka with significant validity and reliability.

There has been limited action to develop and test suitable scales to address the psychological aspect of LF in Sri Lanka. The national PELF had already implemented a programme MMDP to address the physical issues of LF morbidity. In the same MMDP programme, mental health aspects were addressed, but without proper guidelines. Therefore, development of a new tool to measure the psychosocial burden in LF patients in the country is an urgent need $(17,24)$. It was proposed that the use of support groups had a role in the lymphoedema management. Patient support groups in Haiti, and Dreyer's 'Hope Clubs' in Brazil have shown to improve the quality of life (QOL) among lymphoedema patients, also provide ongoing social and emotional support $(12,25)$.

In contrast, in the Sri Lankan study, many of the older interviewees would be willing to attend community gatherings with local lymphoedema patients to discuss their problems. However, the younger subjects wished to keep to themselves, and those who had difficulty in walking or standing were generally reluctant to participate at gatherings (11). Additionally, some patients in a recent qualitative study expressed that interviews themselves were a therapeutic release, which suggested that talking may be beneficial in some context (8). These suggestions revealed that psychological practices for one country might not be suitable in a different cultural context.

Three (03) important tools were identified; Disability Assessment Schedule 2.0 (WHODAS 2.0), the Dermatology Life Quality Index (DLQI), and the Lymphatic Filariasis Quality of Life Questionnaire (LFSQQ). DLQI was translated to Sinhala language and tested in samples of Sri Lankan populations $(11,20)$ however, noticed that the questionnaires were not covering the dimensions we were expecting. The Sinhala translation of
WHODAS 2.0 is currently used in one of our ongoing projects with multiple drawbacks. Therefore, the control of psychosocial burden associated with LF in national MMDP programme has been stalled due to non-availability of scientific scale to measure the burden (17). The initial approach to develop a specific scale to assess the psychosocial burden of LF patients through in-depth interviews with patients, has produced an unbiased environment to collect reliable information (19).

Forty-six (46) in-depth interviews were an adequate sample for a qualitative study. Well planned indepth interviews assisted the interviewer, transcriber and translator to maintain the standards needed in the scale development process by preventing, even losing patient's vague expressions $(26,27)$. PSB-CL scale was developed using the methodology described in quality of life measurement scale development (19). However, in the psychometric analysis, we could not perform advanced analysis by using Classical Test Theory (CTT) and Item Response Theory (IRT) (Rasch analysis) due unavailability of software. We abled to do only principal component analysis with SPSS by producing significant results (Table 3). Thus, this PBS-CL scale promises to be a good tool to measure the psychosocial burden of LF patients, and appears to have psychometric properties at a reasonable level.

It is noteworthy that the patient's problems and suffering that arise from time to time cannot be measured with $100 \%$ accuracy by using a dimensional model. There is a clear distinction between cases and non-cases with categorical approach, but not with the dimensional (19). In the process of dimensional model development, loss of pieces of vital information is inevitable, and may have a considerable effect on the overall content and structure of the questionnaire. However, there are advantages in the dimensional approach over the categorical approach. In the categorical approach, it is difficult, especially in psychosocial assessments, to have more than one problem, but the dimensional approach does permit this; some problems may be present, present in a mild form, even problems can coexist (19). 
The transformation of speaking language to a written language, and using this as a direct or indirect questionnaire may not be the best option to measure psychosocial problems in some patients. The psychological and social burden of people in a developing country is due to various reasons, and they are embedded in the population for a longer period (28). Unfortunately, the reasons are more complex and originated from various dimensions like economic problems, social conflicts and the thinking pattern derived from the culture and religion. Further, we observed that the other common diseases like diabetes, hypertension, eczemas, varicose veins etc. have caused psychosocial problems in LF patients and interfered with the LF related psychosocial burden.

The meaning lost in audio transcribing became an information barrier. According to the selected methodology, the patients' minor facial expressions of emotional gestures could not be documented during the interview $(26,27)$. Even though we tried to transcribe patients' emotional variation of voice, facial expressions and emotional body gestures, it was very difficult with audio recording methodology. However, the best possible representation and understanding of the interpreted experience of the participants and thereby validating qualitative research could overcome these issues to a certain extent (26).

Thus, in PSB-CL scale development process, careful selection of patients for in-depth interviews and item reduction techniques made the final questionnaire more stable and comprehensible. Explorative factor analysis and item analysis helped us to make a final version with meaningful and acceptable factors. The Sinhala questionnaire of PBS-CL has been translated into English by two independent translators for the publication purpose. We are in the process of validating the 15 item PSB$\mathrm{CL}$ questionnaire. We need to use a bigger and representative sample before adopting it as a scale to monitor the psychosocial burden of patients in community settings.

\section{References}

1. Scott AL. Lymphatic-dwelling Filariae. In: Nutman, T.B. ed., Lymphatic Filariasis. London: Imperial College Press; 2000: 5-39.
2. World Health Organization. Global programme to eliminate lymphatic filariasis: progress report, 2018. Weekly Epidemiological Record, 2019;94(41): 457-472.

3. Dreyer G, Dreyer P, Piessens WF. Extralymphatic disease due to bancroftian filariasis. Brazilian Journal of Medical and Biological Research, 1999; 32: 1467-1472.

4. Kumaraswami V, Nutman T. The clinical manifestations of lymphatic filariasis. In: Nutman, T.B. ed., Lymphatic Filariasis. London: Imperial College Press; 2000: 103-125.

5. Addiss DG, Brady MA. Morbidity management in the Global Programme to Eliminate Lymphatic Filariasis: A review of the scientific literature. Filaria Journal, 2007; 6: 2 .

6. Mathew CG, Chu BK, English M, Ottesen EA, Bradley MH, Turner HC. The health and economic burden of lymphatic filariasis prior to mass drug administration programmes. Clinical Infectious Diseases, 2019; e-publication - DOI: 10.1093/cid/ciz671.

7. Weerasooriya MV, Weerasooriya TR, Gunawardena NK, Samarawickrema WA, Kimura E. Epidemiology of bancroftian filariasis in three suburban areas of Matara, Sri Lanka. Annals of Tropical Medicine \& Parasitology, 2001; 95: 263-273.

8. Perera M, Whitehead M, Molyneux DH, Weerasooriya MV, Gunatilleke G. Neglected patients with a neglected disease? A qualitative study of lymphatic filariasis. PLoS Neglected Tropical Diseases, 2007; 1: e128.

9. Chandrasena TN, Premaratna R, De Silva NR. Lymphoedema management knowledge and practices among patients attending filariasis clinics in the Gampaha District, Sri Lanka. Filaria Journal, 2004; 3;3(1): 6.

10. Wijesinghe RS, Wickremasinghe AR, Ekanayake S, Perera MS. Efficacy of a limb-care regime in preventing acute adenolymphangitis in patients with lymphoedema caused by bancroftian filariasis, in Colombo, Sri Lanka. Annals of Tropical Medicine \& Parasitology, 2007; 101: 487-497.

11. Yahathugoda TC, Wickramasinghe D, Weerasooriya MV, Samarawickrema WA. Lymphoedema and its management in cases of lymphatic filariasis: the current situation in three suburbs of Matara, Sri Lanka, before the introduction of a morbidity-control programme. Annals of Tropical Medicine \& Parasitology, 2005; 99: 501-510.

12. Dreyer G, Addiss DG, Dreyer P, Norões J. Basic lymphoedema management: treatment and prevention of problems associated with lymphatic filariasis. Hollis, NH: Hollis Publishing Company; 2002: 112. 
13. World Health Organization. Surgical approaches to the urogenital manifestations of lymphatic filariasis. 2002: WHO/CDS/CPE/CEE.

14. World Health Organization. Lymphatic filariasis: progress of disability prevention activities. Weekly Epidemiological Record, 2004; 79: 417-424.

15. Litt E, Baker MC, Molyneux DH. Neglected tropical diseases and mental health: a perspective on comorbidity. Trends in Parasitology, 2012; 28(5): 195-201.

16. Ton TG, Mackenzie C, Molyneux, DH. The burden of mental health in lymphatic filariasis Infectious Diseases of Poverty, 2015; 4: 34. DOI 10.1186/s40249-015-0068-7

17. Chandrasena TN, Premaratna R, Gunaratna IE, De Silva NR. Morbidity management and disability prevention for lymphatic filariasis in Sri Lanka: Current status and future prospects. PLoS Neglected Tropical Diseases, 2018; 10: 12(5).

18. Wijesinghe RS, Wickremasinghe AR, Ekanayake S, Perera MS. Physical disability and psychosocial impact due to chronic filarial lymphoedema in Sri Lanka. Filaria Journal, 2007; 6: 4.

19. Streiner D, Norman G, Cairney J. Health Measurement Scales: A practical guide to their development and use, 2014; Oxford, UK: Oxford University Press. Retrieved 31 Mar. 2020, from https://oxfordmedicine.com/view.

20. Chandrasena TN, Premaratna R, Muthugala MA, Pathmeswaran A, de Silva NR. Modified Dermatology Life Quality Index as a measure of quality of life in patients with filarial lymphoedema. Transactions of The Royal Society of Tropical Medicine and Hygiene, 2007; 101(3): 245-9.
21. Auerbach CF, Silverstein LB. Qualitative studies in psychology. Qualitative data: An introduction to coding and analysis. New York University Press: USA, 2003.

22. Nevo B. Face validity revised. Journal of Educational Measurement, 1985; 22: 287-193.

23. Cronbach LJ. Coefficient alpha and internal structure of tests. Psychometrika, 1951; 16:297-334.

24. World Health Organization. Global programme to eliminate lymphatic filariasis: Managing morbidity and preventing disability. 2013; Geneva: WHO/WHO/HTM/ NTD/PCT/2013.7

25. Coreil J, Mayard G, Louis-Charles J, Addiss DG. Filarial elephantiasis among Haitian women: social context and behavioural factors in treatment. Tropical Medicine \& International Health, 1998; 3: 467-473.

26. Fenna van N, Tineke A, Hans J, Dorly D. Language differences in qualitative research: Is meaning lost in translation?. European Journal of Ageing, 2010; 7: 313316.

27. Temple B, Young A. Qualitative research and translation dilemmas. Qualitative Research, 2004; 4(2): 161-178.

28. Minas H, Mendis J, Hall T. Mental health in Asia and the Pacific: Historical and Cultural Perspectives, 2017. DOI: 10.1007/978-1-4899-7999-5_4. Access on 2020.01.10. 